\title{
Molecular Mechanism of Light-Induced Acceleration of the Adenosine Triphosphate Conversion to the Cyclic Adenosine Monophosphate Catalyzed by the Photoactivated Adenylyl Cyclase bPAC
}

\author{
Maria G. Khrenova, *a,b Anna M. Kulakova, ${ }^{\text {a }}$ Alexander V. Nemukhin *,a,c
}

${ }^{a}$ Department of Chemistry, Lomonosov Moscow State University, Moscow, 119991, Russian Federation

b Bach Institute of Biochemistry, Federal Research Centre "Fundamentals of Biotechnology" of the Russian Academy of Sciences, Moscow, 119071 Russian Federation

${ }^{c}$ Emanuel Institute of Biochemical Physics, Russian Academy of Sciences, Moscow, 119334, Russian Federation

* Corresponding authors:

Dr. Maria G. Khrenova, Lomonosov Moscow State University, Russian Federation Email: khrenova.maria@gmail.com

Prof. Alexander V. Nemukhin, Lomonosov Moscow State University, Russian Federation Email: anem@lcc.chem.msu.ru 


\begin{abstract}
We report the first computational characterization of an optogenetic system composed of two

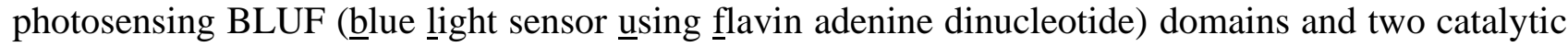
adenylyl cyclase (AC) domains. Conversion of adenosine triphosphate (ATP) to cyclic adenosine monophosphate (cAMP) and pyrophosphate (PPi) catalyzed by ACs coupled with excitation in photosensing domains has emerged in the focus of modern optogenetic applications because of the request in photoregulated enzymes to modulate cellular concentrations of signaling messengers. The photoactivated adenylyl cyclase from the soil bacterium Beggiatoa sp. (bPAC) is an important model showing considerable increase of the ATP to cAMP conversion rate in the catalytic domain after the illumination of the BLUF domain. The $1 \mu$ s classical molecular dynamics simulations reveal that the activation of the BLUF domain leading to tautomerization of Gln49 in the chromophore binding pocket results in switching of position of the side chain of Arg278 in the active site of AC. Allosteric signal transmission pathways between Gln49 from BLUF and Arg278 from AC were revealed by the dynamical network analysis. The Gibbs energy profiles of the ATP $\rightarrow$ cAMP + PPi reaction computed using QM(DFT( $\omega$ B97X-D3/6-31G**))/MM(CHARMM) molecular dynamics simulations for both Arg278 conformations in AC clarify the reaction mechanism. In the light-activated system, the corresponding arginine conformation stabilizes the pentacoordinated phosphorus of the $\alpha$-phosphate group in the transition state, thus lowering the activation energy. Simulations of the bPAC system with the Tyr7Phe replacement in BLUF demonstrate occurrence of both arginine conformations in an equal ratio, explaining the experimentally observed intermediate catalytic activity of the bPAC-Y7F variant as compared with the dark and light states of the wild type bPAC.
\end{abstract}




\section{Introduction}

Photoreceptor protein domains coupled with enzyme domains form powerful light-regulated biocatalysts utilized in optogenetics. ${ }^{1,2}$ Recently, the photoregulated reaction of conversion of adenosine triphosphate (ATP) to cyclic adenosine monophosphate (cAMP) and pyrophosphate (PPi), illustrated in Fig. 1, has emerged in the focus of modern optogenetic applications as a tool to modulate cellular concentration of signaling messengers. ${ }^{3-5}$

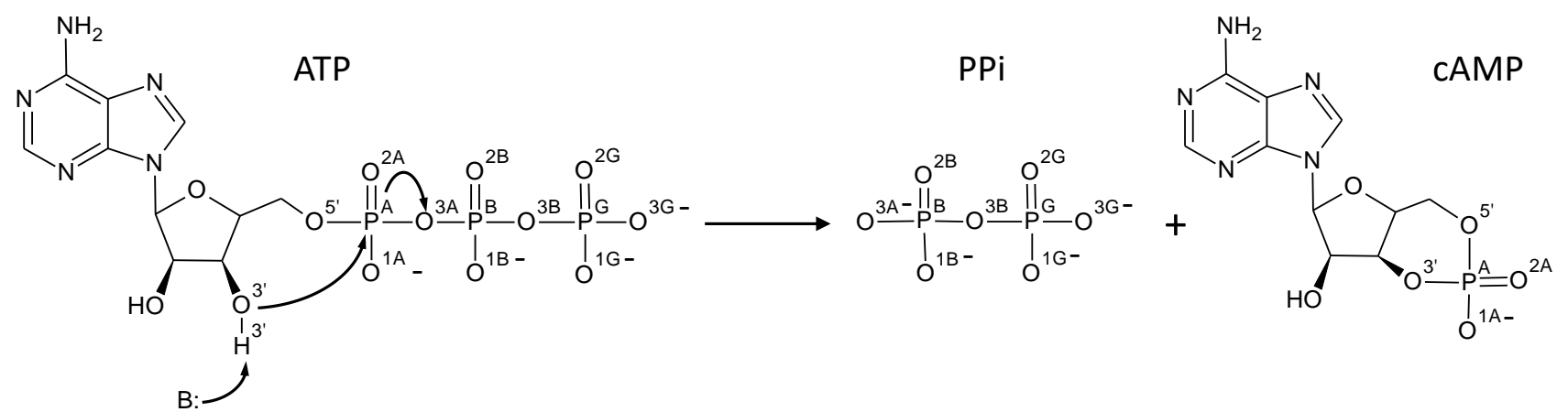

Figure 1. Reaction of the ATP to cAMP conversion catalyzed by adenylyl cyclases. Names of atoms are given according to the IUPAC recommendations. ${ }^{6}$

This reaction catalyzed by adenylyl cyclases (AC) is one of the most important biochemical transformations by itself. ${ }^{7-9}$ It seems deceptively simple: the $\mathrm{P}^{\mathrm{A}}-\mathrm{O}^{3 \mathrm{~A}}$ bond in ATP is cleaved and the $\mathrm{P}^{\mathrm{A}}-\mathrm{O}^{3^{\prime}}$ bond is formed, thus resulting in the cAMP and the pyrophosphate (PPi) reaction products; however, mechanistic details are important, as discussed below.

The AC domains combined with the photosensing domains comprise photoregulated adenylyl cyclases. In particular, the BLUF (blue light sensor using flavin adenine dinucleotide), ${ }^{10-18}$ rhodopsin ${ }^{19}$ and cyanobacteriochrome ${ }^{20}$ photoreceptor proteins are considered as photosensing partners in optogenetic systems. In this work, we focus on the photoactivated adenylyl cyclase (PAC) from the soil bacterium Beggiatoa sp. (bPAC), ${ }^{14,16}$ because it shows a remarkable acceleration of the $\mathrm{ATP} \rightarrow \mathrm{cAMP}$ reaction in the catalytic domains upon light illumination of the photosensing BLUF domains. According to Refs 15,16 , the photoactivation results in the $\sim 300$ fold increase of the ATP to cAMP conversion rate as compared with only up to 20 times photoinduced acceleration in other PACs. ${ }^{15,18}$

The molecular mechanism of the light-induced acceleration of the ATP $\rightarrow$ cAMP chemical reaction in PACs remains uncovered. Lindner et al. ${ }^{15}$ reported the results of structural, kinetic and mutational studies in bPAC, which were interpreted on the base of the obtained crystal structures of the protein in the dark and light-illuminated (shortly, light) states of the BLUF domains containing 
flavin mononucleotide (FMN) cofactors, but bearing no substrates or substrate analogs in the AC domains. These results ${ }^{15}$ are consistent with those obtained in studies of the homologues OaPAC protein ${ }^{12,13,21}$ in respect that small structural changes occur upon dark-to-light transition. The authors of Ref 15 assumed that highly conservative residues of BLUF domain, Tyr7 and Gln49, the kink in $\beta 4_{\mathrm{BLUF}}$ and the C-terminal BLUF capping helix were involved in the signaling pathway. Stierl et al. ${ }^{22}$ described the role of site-directed mutations within the BLUF domain in bPAC. In particular, the protein complex called bPAC-Y7F (i.e., that with the Tyr7Phe mutation) results in locking the BLUF domain in the so-called pseudolit state. ${ }^{23}$ The bPAC-Y7F shows intermediate catalytic activity of the AC domain, as compared with the dark and light states of the wild type bPAC. ${ }^{15,22}$

Importantly, the authors of all papers on PACs ${ }^{12,13,15,22}$ focused almost entirely on the processes in the light-sensitive BLUF domain, but not on the mechanism of chemical reactions of the ATP to cAMP conversion in the catalytic domain. The only available information on the ATP $\rightarrow$ cAMP + PPi reaction in bPAC is the steady state catalytic rate constant ${ }^{14,15}$ and the $\mathrm{K}_{\mathrm{M}}$ value. ${ }^{14}$ The reported rate constant in the dark state is $0.008 \pm 0.001 \mathrm{~s}^{-1}$, whereas it increases to $2.6 \pm 0.3 \mathrm{~s}^{-1}$ under constant blue light illumination. ${ }^{15}$ In this respect, studies of the structurally similar mammalian adenylyl cyclases (mACs) can provide additional data. Ref 24 reported the rate of the chemical reaction inside the protein matrix $59 \mathrm{~s}^{-1}$ following the fitting of rate constants in a complex kinetic model of mAC inhibition. For the goals of our work, it is important to note that Ref 25 emphasized the effect of the conserved arginine residue $\operatorname{Arg} 1029$ in mAC (analogous to Arg278 in bPAC). It was shown that its mutation to Ala resulted in more than 10-fold decrease of the reaction rate, keeping the catalytic activity. ${ }^{25}$ At the same time, this replacement did not affect the binding affinity, i.e. did not change the $\mathrm{K}_{\mathrm{M}}$ value. ${ }^{25}$ The X-ray study (PDB ID: $1 \mathrm{CJK}^{25}$ ) demonstrated that the arginine side chain was located closely to the $\alpha$-phosphate group and might stabilize the reaction transition state. ${ }^{25}$ Following these findings, Linder et al. ${ }^{15}$ tentatively assumed that the corresponding residue Arg278 in the catalytic domain in bPAC might play a role. There evidences demonstrate a very limited knowledge on the mechanism of the ATP to cAMP conversion in bPAC.

Computer simulations can deepen the understanding of molecular processes both in photosensing and catalytic domains. In particular, details of transformations upon light excitation in the BLUF domains originally revealed in simulations ${ }^{26}$ and confirmed later ${ }^{27-31}$ show that the side chain of the conserved glutamine (like Gln49 in bPAC) in the chromophore-containing pocket undergoes amide-imide tautomerization. Mechanism of the ATP $\rightarrow$ cAMP conversion in the active sites of adenylyl cyclases, including mammalian and bacterial ACs unbound to other protein domains, 
was modeled in infrequent computational papers. ${ }^{32-35}$ The latter works employed different versions of the quantum chemistry and quantum mechanics/molecular mechanics (QM/MM) approaches to characterize the reaction route from reactants to products (Fig. 1) in the active sites of the corresponding enzymes, showing somewhat diversity in conclusions. No theoretical simulations revealing the mechanism of photoactivation of catalysis in AC have been reported for bPAC, as well as for any other PAC systems.

The aim of this work is to establish the atom level mechanism of the ATP to cAMP conversion in bPAC and to reveal the origin of the acceleration of this reaction upon blue-light illumination of the protein complex. After construction of an all-atom model molecular system of bPAC, we apply modern approaches based on molecular dynamics (MD) simulations. We recognize conformational changes in this optogenetic complex following transition from the dark to the light states and identify the key role of switching between conformations of the Arg278 side chain in the AC active site. We find that this switching is important in the wild-type bPAC and in its bPAC-Y7F variant with an intermediate catalytic activity. We compute and compare the Gibbs energy profiles of the ATP $\rightarrow$ cAMP reaction in the dark and light states of bPAC using MD with the quantum mechanics/molecular mechanics $\left(\mathrm{QM}\left(\mathrm{DFT}\left(\omega \mathrm{B} 97 \mathrm{X}-\mathrm{D} 3 / 6-31 \mathrm{G}^{* *}\right)\right) / \mathrm{MM}\right)$ potentials. We also consider qualitative aspects of the chemical reaction in this protein environment by analyzing the relevant electron density based descriptors. ${ }^{36-38}$

The outline of the paper is as follows. After methodology section, we describe the construction of model systems and the results of large-scale classical MD simulations in both dark and light states of the wild type bPAC and bPAC-Y7F mutant followed by the dynamical network analysis that clarifies the signal transmission path from the flavin chromophore in BLUF to Arg278 in the AC domain. Next, we describe the results obtained with the ab initio type QM/MM MD calculations of the Gibbs energy profiles in the dark and light states. This allowed us to formulate for a first time a consistent picture of photoexcitation and enzymatic processes in bPAC.

\section{Methods}

The crystal structure (PDB ID: 5M2A ${ }^{15}$ ) of the bPAC dimer with the FMN cofactors in the photoreceptor domains, but without substrate or substrate analogs in the catalytic domains, was used as a source of initial coordinates of heavy atoms. Amino acid residues, which were poorly resolved in the X-ray analysis, were added using the data on the primary sequence (UniProt A7BT71). Hydrogen atoms were added using the Reduce program ${ }^{39}$ to reproduce the protonation states of amino acids at 
neutral $\mathrm{pH}$. The ATP molecule was manually added to each subunit of bPAC following motifs of the crystal structures of complexes of human AC with ATP (PDB ID: $4 \mathrm{USW}^{40}$ ) and with the guanosine triphosphate (GTP) analog GTP $\gamma \mathrm{S}$ (PDB ID: $1 \mathrm{CJK}^{25}$ ). Thus constructed model was assigned to the dark state of the protein complex. The light state of bPAC was obtained from the dark state by replacing the amide group of the Gln49 side chain by the imide group, i.e. assuming tautomerization of the Gln49 side chain. ${ }^{26,27,31}$ The bPAC-Y7F mutant was constructed from the dark state model system replacing Tyr7 by phenylalanine. All these model systems were solvated in a rectangular water box with the dimension of $111 \times 97 \times 130 \AA^{3}$ and properly neutralized. Despite the large system size we utilized all-atom force fields: CHARMM36 ${ }^{41,42}$ for the protein, ATP and the magnesium ions, the TIP3P $^{43}$ for water molecules. Force field parameters for FMN molecule were obtained from the CGenFF server ${ }^{44}$ and additionally optimized. ${ }^{45}$ Parameters of the imide form of glutamine were taken from our previous work. ${ }^{46}$

The VMD software ${ }^{47}$ was used for preparation of all considered systems and for analysis of molecular dynamics (MD) trajectories. All MD calculations were carried out with a 1 fs integration time step using NAMD 2.13 software package. ${ }^{48}$ The NPT ensemble was utilized with the $\mathrm{P}=1 \mathrm{~atm}$ and $\mathrm{T}=300 \mathrm{~K}$ with the pressure and temperature control by Nosé-Hoover barostat and Langevin thermostat, respectively. The cutoff distances were $12 \AA$ for both electrostatic and van der Waals interactions with switching to the smoothing function at $10 \AA$.

Preparation of bPAC complexes for the production runs consisted of two steps: equilibration of solvating water shells and equilibration of the entire system. At the first step, the $1 \mathrm{~ns}$ molecular dynamic simulation was performed keeping the coordinates of the protein, magnesium ions, ATP and FMN fixed. The second step was a $20 \mathrm{~ns}$ MD run with no constraints or restraints. The representative frame from the last $5 \mathrm{~ns}$ of this trajectory was used to select coordinates for further MD simulations. The total length of the production runs was $1 \mu$ s for each model. To avoid accumulation of the computational errors and to gain better sampling of the conformational space, we executed sets of the trajectories with tens of nanoseconds each as suggested in Ref ${ }^{49}$.

Dynamical network analysis ${ }^{50}$ was utilized to dissect the signaling pathways from photosensing to catalytic domains in the bPAC dimer. According to this approach, a network is defined as a set of nodes connected by edges. In this work, every amino acid was represented by a single node; the ATP and FMN moieties were divided into two nodes each. Any two nodes (except the neighbors) were connected by an edge if the distance between them was less than $4 \AA$ for more 
than $75 \%$ of the simulation time. Covariance and correlation matrices for dynamical network analysis were calculated with the Carma program. ${ }^{51}$

The QM/MM MD simulations with the ab initio type potentials in QM were performed to study the reaction mechanism in the dark and light states. We exploited the NAMD program for MD calculations, and the NAMD interface ${ }^{52}$ between the TeraChem program ${ }^{53}$ for calculating forces in QM and NAMD program for calculating forces in MM. ${ }^{48}$ The starting points for the QM/MM MD simulations were obtained from the representative frames of the classical MD trajectories of the dark and light states. The QM subsystem was described at the $\omega$ B97X-D3/6-31G** Kohn-Sham DFT level. The rest of the system, i.e. the MM part, was described with the same force field parameters as in the classical MD simulations. The 5 ps QM/MM MD pre-equilibration of the ES (enzyme-substrate) complexes was performed. Then, the 10 ps production runs of the ES complexes were executed. The ES to EP (enzyme-product) transition was calculated within the umbrella sampling approach followed by the umbrella integration analysis. The reaction coordinate, $\xi$, was set as a difference of two distances, $\mathrm{d}\left(\mathrm{O}^{3^{\prime}} \ldots \mathrm{P}^{\mathrm{A}}\right)$ and $\mathrm{d}\left(\mathrm{P}^{\mathrm{A}} \ldots \mathrm{O}^{3 \mathrm{~A}}\right)$ being positive in the $\mathrm{ES}$ and negative in EP regions. The force constant, $\mathrm{K}$, of the harmonic potential $\mathrm{K} \cdot\left(\xi-\xi_{0}\right)^{2}$ was set to $40 \mathrm{kcal} / \mathrm{mol}$. These potentials were centered at the respective $\xi_{0}$ values with the $0.3 \AA$ increment with the ranges depending on the model system. First $0.5 \mathrm{ps}$ from each MD run was excluded from the statistical analysis. Finally, the $10 \mathrm{ps}$ runs for each EP complexes were performed.

The Laplacian of the electron density, $\nabla^{2} \rho(\mathbf{r}),{ }^{36,54}$ was calculated at different frames of the QM/MM MD trajectory of the ES complex to discriminate reactive and nonreactive species. Previous works $37,38,55,56$ demonstrate that this approach is a proper tool to visualize the activation in the nucleophilic attack step. In the molecular systems the areas of the local electronic charge concentration regions with $\nabla^{2} \rho(\mathbf{r})<0$ (electrophilic sites) and electronic charge depletion areas with $\nabla^{2} \rho(\mathbf{r})>0$ (nucleophilic sites) are formed. Analysis of $\nabla^{2} \rho(\mathbf{r})$ in the plane formed by the nucleophilic $\mathrm{O}^{3^{\prime}}$ atom and the $\mathrm{P}^{\mathrm{A}}-\mathrm{O}^{3 \mathrm{~A}}$ bond provides easily visible images, which help to discriminate conformations favorable and unfavorable for the chemical reaction. Namely, electron lone pair on the $\mathrm{O}^{3^{\prime}}$ atom is oriented towards the electrophilic $\mathrm{P}^{\mathrm{A}}$ if the ATP is prepared for the chemical reaction. Electron density analysis was performed in the Multiwfn program package. ${ }^{57}$ 


\section{Results and Discussion}

\section{Computationally derived structures of bPAC}

As described in Methods, we constructed the systems modeling the bPAC dimer composed of two BLUF domains with the FMN molecules in the chromophore-containing pockets and two AC domains containing the ATP molecules. Model systems correspond to the dark (DS) and light (LS) states of the wild-type bPAC and to the bPAC-Y7F variant. A general view on thus obtained complex is illustrated in Fig. 2. The panels in the right side in Fig. 2 show some patterns in the chromophorecontaining pocket in BLUF and in the active site in AC.

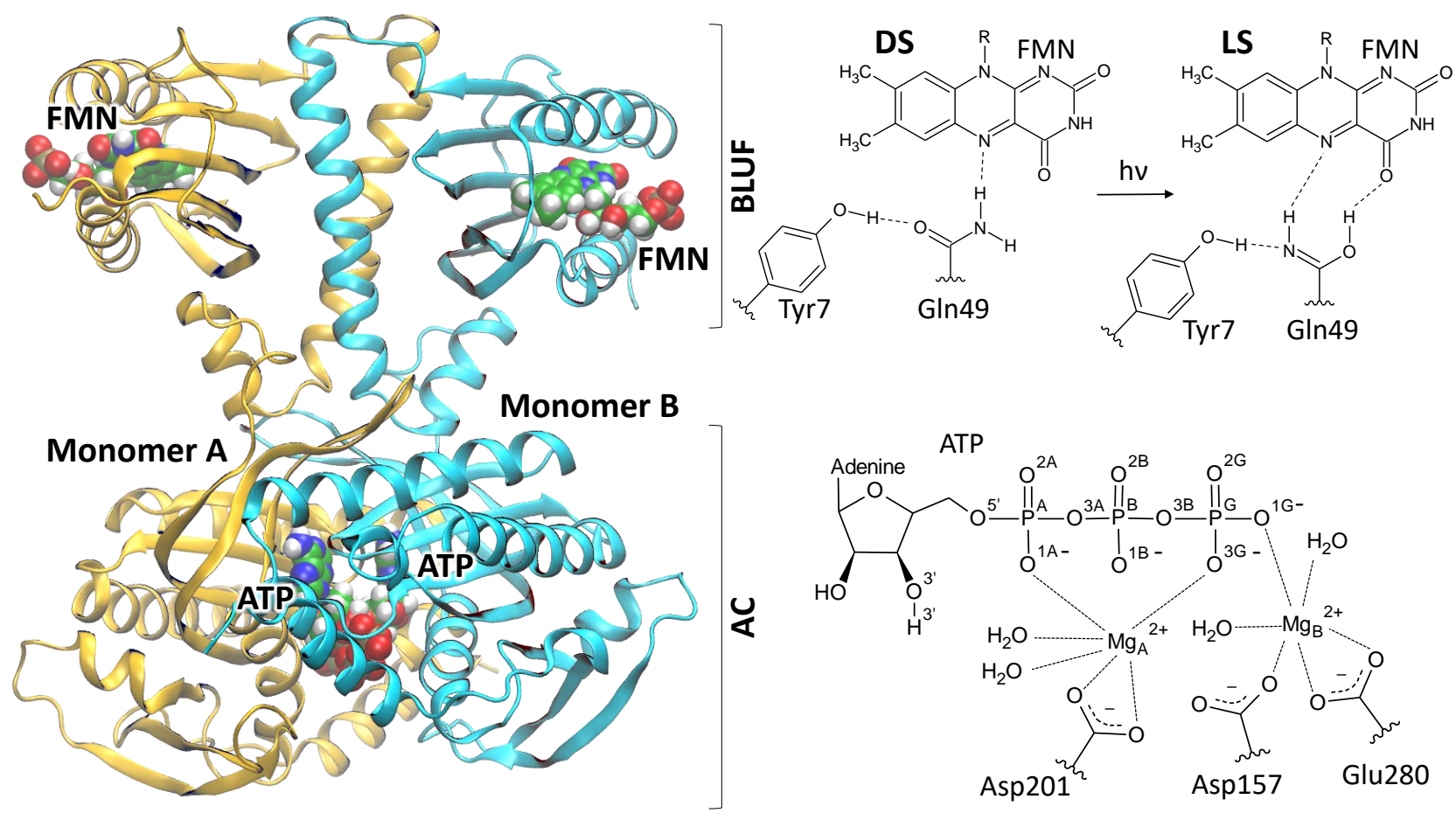

Figure 2. The left side illustrates a general view on the computationally derived bPAC dimer. Monomer A is shown in yellow, monomer B - in cyan. The FMN and ATP molecules are shown as space-filling spheres. Here and in all figures below, the color code is the following: carbon in green, nitrogen in blue, phosphorus in orange, magnesium in magenta, oxygen in red and hydrogen in white. The right side illustrates the photoinduced transformations in the BLUF domain (upper panel) and the active site in $\mathrm{AC}$ (lower panel).

Two important residues Tyr7 and Gln49 are located near the FMN cofactor in BLUF (Fig. 2). In the wild type BLUF, light illumination initiates a photoreaction that comprises the electron and proton transfer to the isoalloxazine ring of the FMN molecule. ${ }^{30}$ After relaxation, the FMN molecule returns to its oxidized state and the proton rearrangement takes place. As a result of this process, the Gln49 side chain tautomerizes and rotates around the single C-C bond (Fig. 2). ${ }^{26,27,31}$ Therefore, in 
our simulations, the dark and light states differ in the conformation of the Gln49 side chain. In the bPAC-Y7F mutant, the photoreaction is locked, and the Gln49 side chain remains in the same amide form independent of illumination. ${ }^{15}$

The active site in AC domains (Fig. 2) carries two magnesium cations, both of them are hexacoordinated with water molecules, the oxygen atoms of ATP and the side chains of the glutamate and aspartate residues, in agreement with the available structures of the mammalian ACs complexed with the substrate or its analogues. ${ }^{25,40}$

We analyzed dynamical behavior of three model systems, DS, LS and bPAC-Y7F, as obtained in the $1 \mu \mathrm{s}$ MD trajectories. We paid the major attention to the individual residues that might be responsible for the changes of the ATP $\rightarrow$ cAMP reaction rate, i.e. to those located in the active site of $\mathrm{AC}$ or in the immediate vicinity of the latter. The most important conclusion refers to the Arg 278 conformations, which differ significantly in the considered model system. This means that the arginine side chain adopts two different conformations in the dark and light states of the complex. Distributions of the $\chi$ dihedral angle (see Fig. 3), corresponding to the $\mathrm{C}^{\mathrm{A}}-\mathrm{C}^{\mathrm{B}}-\mathrm{C}^{\mathrm{G}}-\mathrm{C}^{\mathrm{D}}$ fragment of the arginine side chain, allow us to characterize these conformations. In DS (blue line in Fig. 3), values of $\chi$ are predominantly negative with the distribution maximum located at around $-65^{\circ}$. In LS (red line in Fig. 3 ), this population is minor, and the vast majority of conformations correspond to the $\chi$ values around $70^{\circ}$. DS-type conformations with the negative $\chi$ values promote the hydrogen bond interaction between the guanidinium group of $\operatorname{Arg} 278$ and the $\mathrm{O}^{1 \mathrm{~B}}$ atom of the ATP $\beta$-phosphate group, as explained in detail below. If $\chi=70^{\circ}$, the side chain of Arg278 interacts with the $\mathrm{O}^{1 \mathrm{~A}}$ atom of the ATP $\alpha$-phosphate group. This conformation should be more favorable for the chemical reaction, because the $\mathrm{P}-\mathrm{O}$ bond cleavage in nucleotide phosphates is normally facilitated by an arginine residue due to stabilization of the transition state and corresponding lowering of activation energy barriers. ${ }^{58}$ The computed distribution for the bPAC-Y7F system (magenta line in Fig. 3) provides further support to the hypothesis of the role of $\operatorname{Arg} 278$ in the photoinduced acceleration of the reaction rate. We see the presence of both arginine conformations in an equal ratio, which is consistent with the experimentally observed ${ }^{15}$ intermediate catalytic activity of the bPAC-Y7F variant as compared with the dark and light states of the wild type bPAC. 


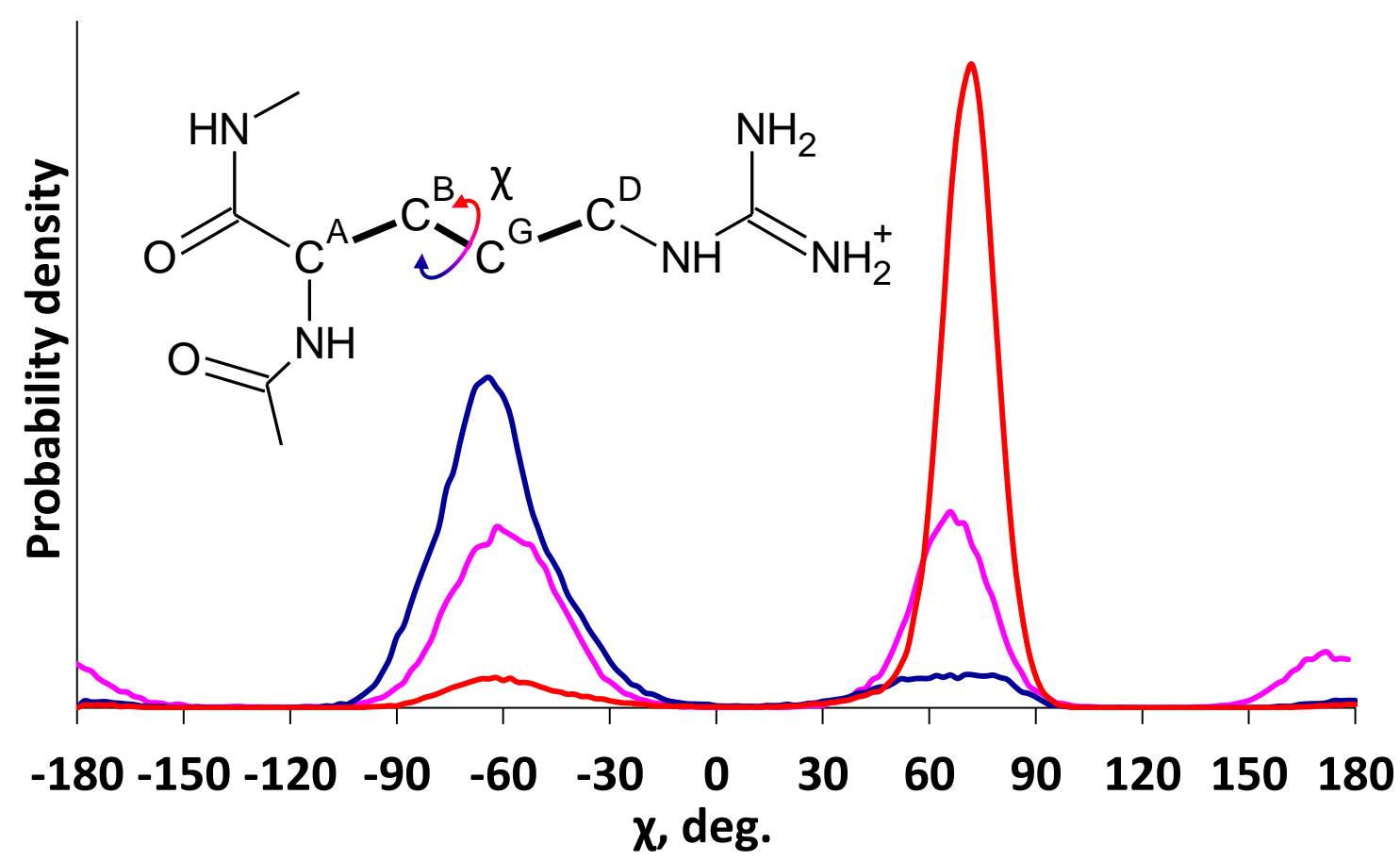

Figure 3. The $\chi$ dihedral angle distributions in the Arg278 side chain along classical MD trajectories in DS (blue), LS (red) and bPAC-Y7F (magenta) model systems.

\section{Signal transmission from the photosensing domain to the catalytic domain}

We analyze allosteric signal transmission pathways from Gln49 to Arg278 in the dark and light states of the wild type bPAC and identify amino acid residues that are involved in the signaling paths more frequently (Fig. 4). The Gln49, playing a critical role in BLUF domain photo-induced processes, is hydrogen bonded to the FMN; its side chain tautomerizes during the photoreaction. ${ }^{26,27,31}$ The signal proceeds to the neighboring $\beta$-sheets, $\beta 1_{\text {BLUF }}$ (Tyr7) and $\beta 4_{\text {BLuF }}$ (Leu75) (Fig. 4). The importance of Tyr7, which is involved in the signaling pathway, is demonstrated in the experimental study on the light regulated AC activity in mutated bPAC variants. ${ }^{15}$ It is in line with other experimental results on the mutational analysis of the BLUF-containing protein PixD. ${ }^{23}$ The Leu75 is a conservative residue among BLUF domains ${ }^{15}$ and its presence in the signal pathway is also reasonable. The signal transmission pathway is different in the bPAC-Y7F variant due to the absence of hydrogen bond interactions between Phe7 and Gln49.

After leaving the BLUF domain, the signal pathway proceeds through Asn136 and Tyr133 from the handle of the protein complex ${ }^{15}$ and reaches the AC domain (Fig. 4). Predominantly, the pathways proceed through the $\beta 5_{\mathrm{AC}}$ (His266) and $\beta 4_{\mathrm{AC}}$ (Val253, Glu255). These $\beta$-sheets form the socalled tongue in the bPAC structure. ${ }^{15}$ According to the X-ray studies, the His266 residue was found 
to be in different conformations in dark and light states that justifies its appearance in the signal pathway. ${ }^{23}$ The $\alpha 4_{\mathrm{AC}}$ (Ala272) is the last intermediate point before reaching Arg278. Thus, computationally derived signal transmission pathways between the Gln49 and Arg278 comprise amino acid residues, which presumably are involved in the signal transduction according to the analysis of the X-ray structures. ${ }^{15}$

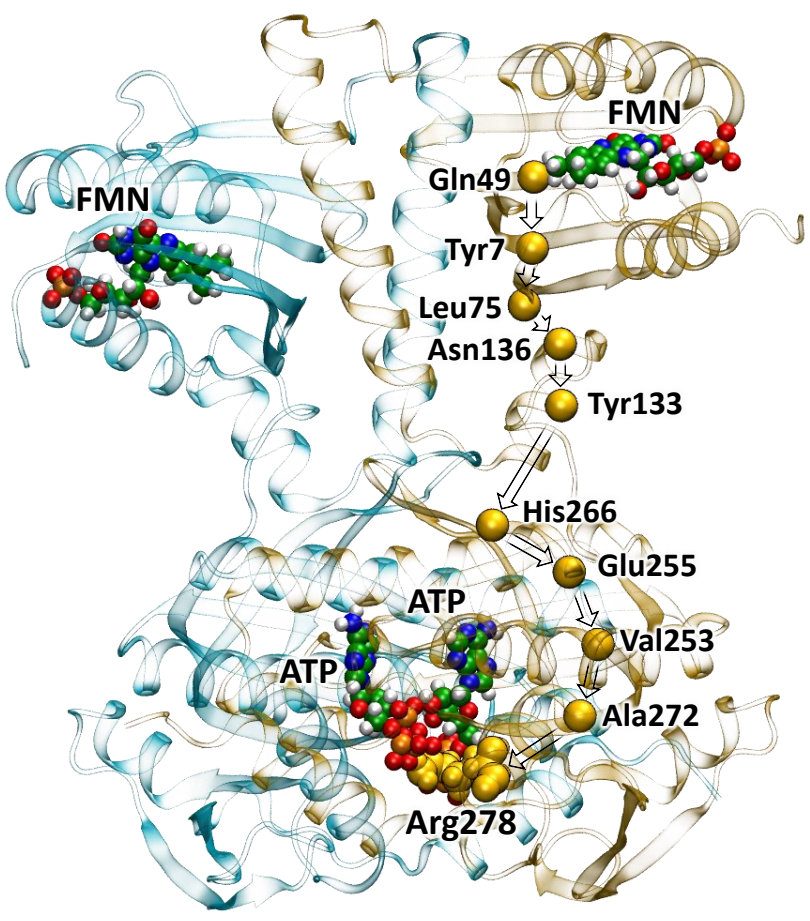

Figure 4. Amino acid residues involved in the signaling path from the Gln49 to the Arg 278 in bPAC. The nodes are shown as yellow balls.

\section{Mechanism of the chemical reaction ATP $\rightarrow$ cAMP in the dark and light states}

According to the classical MD simulations described above, the Arg278 side chain adopts two different conformations near the phosphate groups of ATP in the AC active site in the dark and light states of bPAC. The corresponding structures of the enzyme-substrate complexes, ES-DS and ES-LS, are illustrated in Fig. 5. Initial coordinates of these structures were taken from the frames of classical MD trajectories; then they were employed to launch the QM(DFT $(\omega B$ 97X-D3/631G**))/MM(CHARMM) MD calculations. The guanidine group of Arg278 forms the hydrogen bonds with the oxygen atom of the ATP $\beta$-phosphate group in the ES-DS structure, whereas the Arg278 side chain is located near the $\alpha$-phosphate group and forms the hydrogen bond with the $\mathrm{O}^{1 \mathrm{~A}}$ atom of ATP in the ES-LS structure (Fig. 5). The values of interatomic distances and angles specified 
in Fig. 5 refer to the results of QM/MM MD simulations. They illustrate the pronounced differences of the important geometry parameters in the DS and LS.
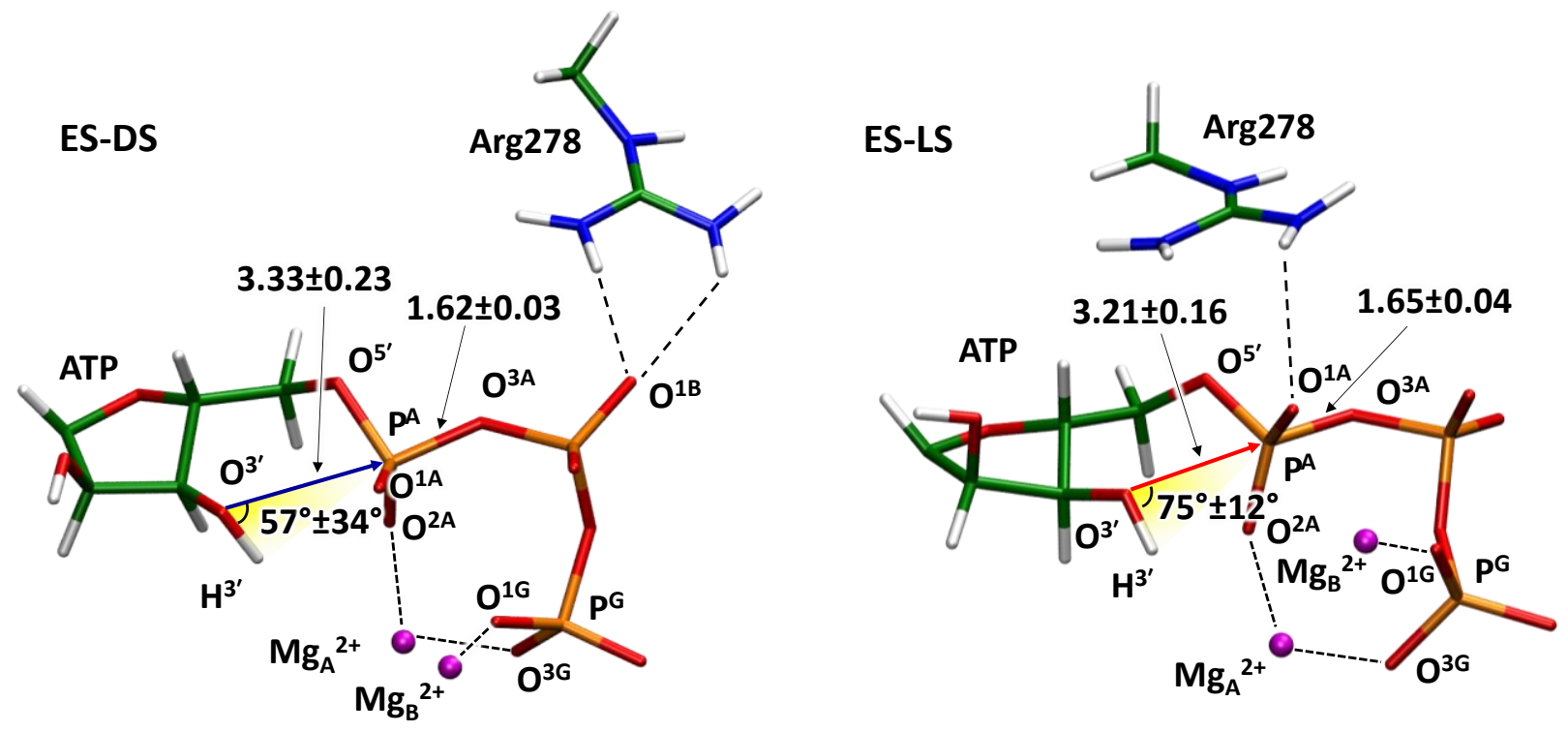

Figure 5. Fragments of the ES complexes in the dark (ES-DS, left) and light (ES-LS, right) states. Distances (in $\AA$ ) and angles refer to the results of QM/MM MD calculations.

A nucleophilic attack of the $\mathrm{O}^{3^{\prime}}$ atom of the ribose group on the $\mathrm{P}^{\mathrm{A}}$ atom following proton transfer from $\mathrm{O}^{3} \mathrm{H}^{3^{\prime}}$ is the key issue in the ATP $\rightarrow$ cAMP reaction in ACs. The upper panel in Fig. 6 shows the distribution of the $\mathrm{O}^{3^{\prime}}-\mathrm{P}^{\mathrm{A}}$ distances in ES-DS (blue line) and ES-LS (red line) obtained in QM/MM MD simulations. In the ES-LS case, this distribution is narrower and it is shifted to smaller values as compared with the distribution in ES-DS. This is a strong indication that the nucleophilic attack should occur more readily in the ES-LS structure. 

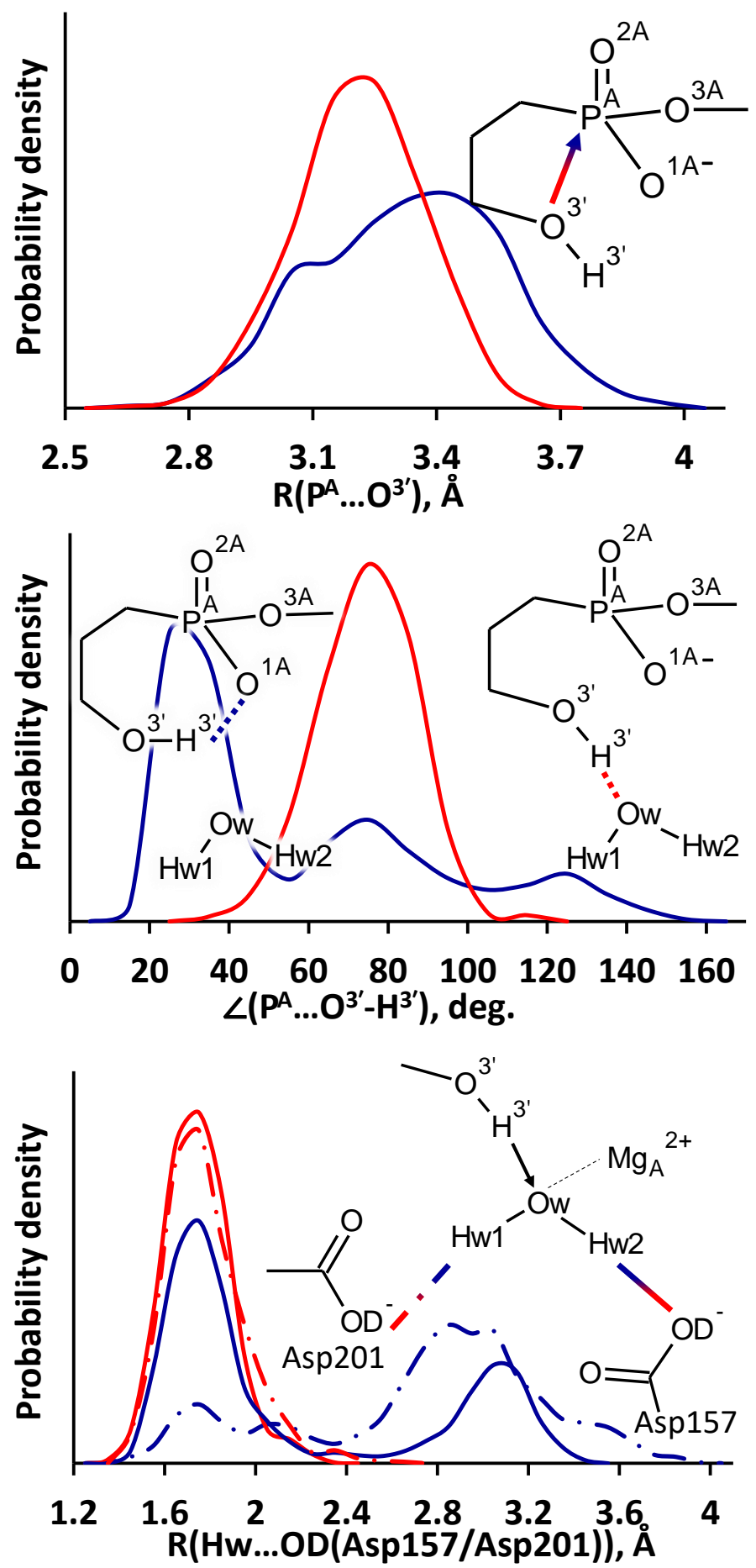

Figure 6. Distributions of geometry parameters in the enzyme-substrate complexes in the dark (ESDS, blue) and light (ES-LS, red) states as obtained in QM/MM MD calculations. The upper panel illustrates the distribution of the distance of the nucleophilic attack. The middle panel shows the distribution of the angle between the $\mathrm{O}^{3^{\prime}}-\mathrm{H}^{3^{\prime}}$ bond and the direction of the nucleophilic attack. The lower panel shows distributions of hydrogen bond lengths between a hydrogen atom (Hw1 or Hw2) of the water molecule, Hw, and the oxygen atoms of the carboxyl group of Asp157 (solid line) and Asp201 (dash-dotted line). 
Two more panels in Fig. 6 illustrate distributions of other geometry parameters in the ES complexes along QM/MM MD trajectories, which are important for the reaction. As we noted in Introduction, previous modeling of AC catalysis ${ }^{32-35}$ using different versions of the QM/MM method do not fully agree in details of the mechanism. Specifically, a candidate for the general base to accept the proton from the ribose group $\mathrm{O}^{3} \mathrm{H}^{3}$ of ATP (see Fig. 1) is indicated differently in previous works. Looking ahead, we claim that in the present simulations, the $\mathrm{H}^{3^{\prime}}$ proton is transferred to Asp157 via a water molecule coordinated by $\mathrm{Mg}_{\mathrm{A}}{ }^{2+}$. This is a mechanism similar to that described in the most recent computational study of the mammalian AC. ${ }^{32}$ Examination of geometry parameters in the ES complexes and analysis of the Laplacian of electron density maps shown in Figure 7 provide a sound support to this reaction pathway.

The middle panel in Fig. 6 illustrates distribution of the $\angle\left(\mathrm{P}^{\mathrm{A}}-\mathrm{O}^{3^{\prime}}-\mathrm{H}^{3^{\prime}}\right)$ angle in ES-DS (blue line) and ES-LS (red line). The distribution for the ES-LS structure shows single maximum at around $80^{\circ}$. In this conformation, $\mathrm{H}^{3^{\prime}}$ is hydrogen bonded to the water molecule that may serve as a proton transfer shuttle to either Asp157 or Asp201 (see Fig. 2). In ES-DS, the corresponding peak is much smaller and the major conformation refers to the value of $\angle\left(\mathrm{P}^{\mathrm{A}}-\mathrm{O}^{3^{\prime}}-\mathrm{H}^{3^{\prime}}\right)$ at around $30^{\circ}$. In this case, $\mathrm{H}^{3^{\prime}}$ forms a hydrogen bond with the $\mathrm{O}^{1 \mathrm{~A}}$ atom. These distributions emphasize an important role of the $\operatorname{Arg} 278$ conformation. In LS, a stable hydrogen bond is formed between $\operatorname{Arg} 278$ and $\mathrm{O}^{1 \mathrm{~A}}$ that prevents formation of the $\mathrm{H}^{3^{\prime}} \ldots \mathrm{O}^{1 \mathrm{~A}}$ hydrogen bond. In $\mathrm{DS}, \mathrm{H}^{3^{\prime}}$ forms a hydrogen bond with either $\mathrm{O}^{1 \mathrm{~A}}$ or with a water molecule along the MD trajectory. Formation of the $\mathrm{H}^{3^{\prime}} \ldots \mathrm{O}^{1 \mathrm{~A}}$ hydrogen bond in ES-DS leads to the following features. First, the distance of the nucleophilic attack increases. Second, a wrong orientation of the electron lone pair on the $\mathrm{O}^{3^{\prime}}$ atom responsible for the nucleophilic attack is observed in the Laplacian of electron density maps (Fig. 7). 

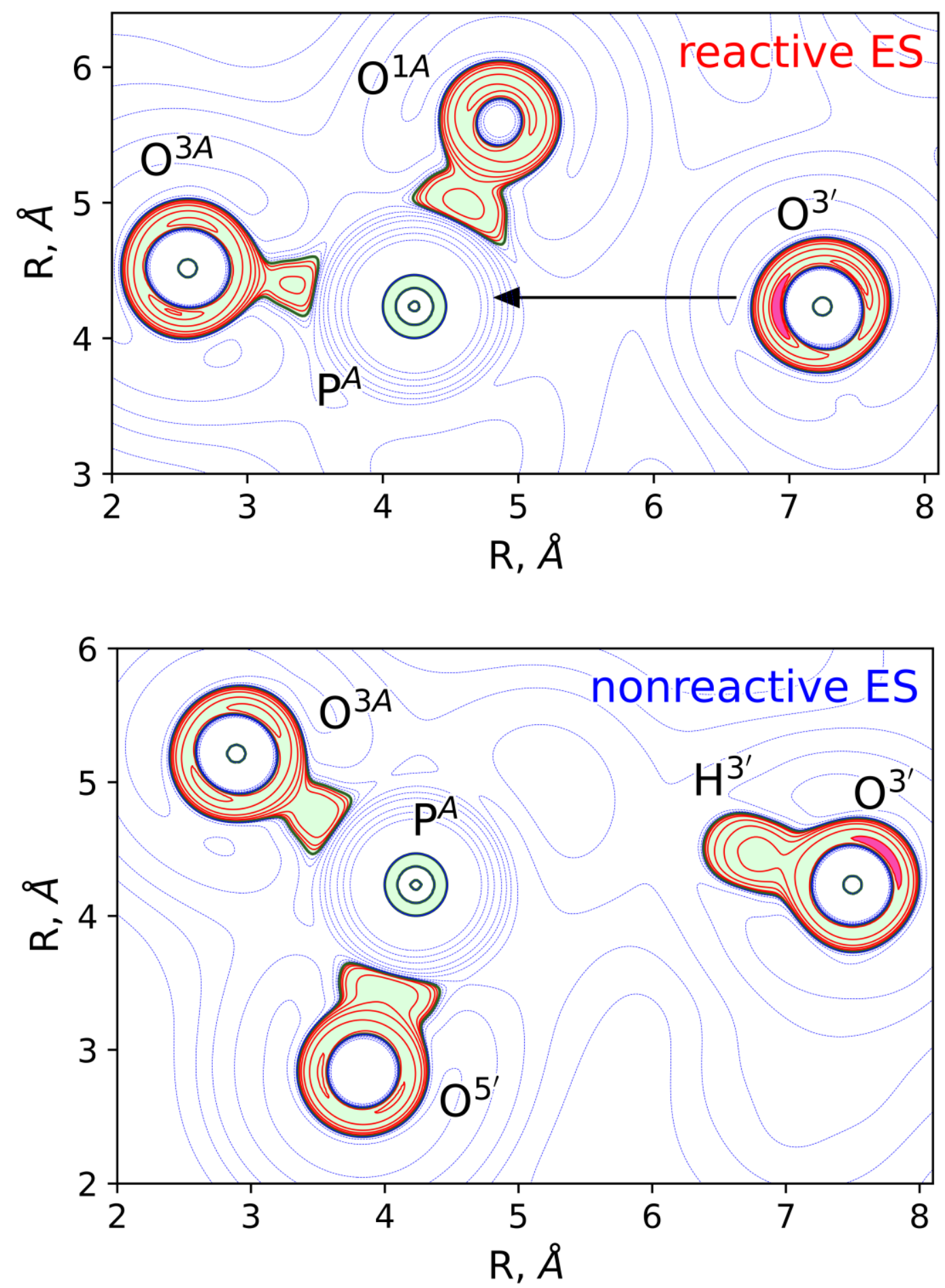

Figure 7. The Laplacian of electron density in the plane formed by the nucleophile, the oxygen atom $\mathrm{O}^{3^{\prime}}$, and the $\mathrm{O}^{3 \mathrm{~A}}-\mathrm{P}^{\mathrm{A}}$ group of ATP. Upper panel shows the graph for the reactive ES; lower panel for the nonreactive ES. Contour lines are $\pm(2 ; 4 ; 8) \cdot 10^{\mathrm{n}}$ au, $-2 \leq \mathrm{n} \leq 1$, blue dashed contour lines indicate the electron density depletion areas $\left(\nabla^{2} \rho(\mathbf{r})>0\right)$ and red solid lines identify the electron density concentration $\left(\nabla^{2} \rho(\mathbf{r})<0\right)$, green solid line corresponds to $\nabla^{2} \rho(\mathbf{r})=0$. The area with $\nabla^{2} \rho(\mathbf{r})<0$ is colored in light green and the lone pair on the oxygen atom $\mathrm{O}^{3^{\prime}}$ is highlighted magenta $\left(\nabla^{2} \rho(\mathbf{r})<-4\right)$. 
If a conformation favors the nucleophilic attack, the electron lone pair in $\mathrm{O}^{3^{\prime}}$ (highlighted in magenta in the upper panel in Fig. 7) is oriented towards the electrophilic $\mathrm{P}^{\mathrm{A}}$ atom. Following the previous studies of nucleophile reactions ${ }^{37,38}$ we denote such complexes as "reactive ES". Contrary, if a $\mathrm{H}^{3^{\prime}} \ldots \mathrm{O}^{1 \mathrm{~A}}$ hydrogen is formed (Fig. 6), the electron lone pair shown in bottom panel in Fig. 7 (highlighted in magenta) is oriented to the opposite side from the $\mathrm{P}^{\mathrm{A}}$ atom. This corresponds to "nonreactive ES" complexes.

Other important geometry parameters favoring the chemical reaction are the hydrogen bonds that facilitate the $\mathrm{H}^{3^{\prime}}$ transfer to one of the aspartates. A water molecule that is hydrogen-bonded to the $\mathrm{H}^{3}$ atom serves as a hydrogen bond shuttle to carboxylates of the aspartate residues, Asp157 and Asp201. From the qualitative side, both of them are candidates to accept a proton during the reaction. We analyze stability of both of these hydrogen bonds (Fig. 6, lower panel). In ES-LS trajectory, both hydrogen bonds are formed during the simulation. In ES-DS, the dynamics is different, the fraction of the frames with both hydrogen bonds is about $5 \%$, whereas only one of the hydrogen bonds is predominantly formed.

Results of these mostly qualitative considerations are perfectly consistent with the conclusions obtained in the straightforward calculations of the reaction energy profiles using the QM(DFT( $\omega$ B97X-D3/6-31G**))/MM(CHARMM) MD simulations. The Gibbs energy profiles shown in Fig. 8 are computed along the reaction coordinate $\xi$ defined as a difference between the $\mathrm{P}^{\mathrm{A}}-$ $\mathrm{O}^{3^{\prime}}$ and $\mathrm{O}^{3 \mathrm{~A}}-\mathrm{P}^{\mathrm{A}}$ distances. It is positive in the ES region and negative in the enzyme-product (EP) region. The ES minima are located at the $\xi$ values of $1.4 \AA$ and $1.5 \AA$ for the LS and DS systems, respectively. The complex reaction coordinate (collective variable) does not provide information on the individual geometry parameters; therefore, we extracted values of the nucleophilic attack distances from the QM/MM MD simulations of the ES complexes. Those are $3.21 \pm 0.16 \AA$ and $3.33 \pm 0.23 \AA$ for the ES-LS and ES-DS, respectively, indicating that the ES complex is tighter in the light state (Fig. 5). The energy barrier of the nucleophilic attack is much lower in LS being $8.4 \mathrm{kcal} / \mathrm{mol}$ as compared with the DS case with the computed barrier of $15.4 \mathrm{kcal} / \mathrm{mol}$. In both pathways, the $\mathrm{H}^{3^{\prime}}$ proton is transferred to a water molecule and the latter transfers its proton to Asp157 (Fig. 9). 

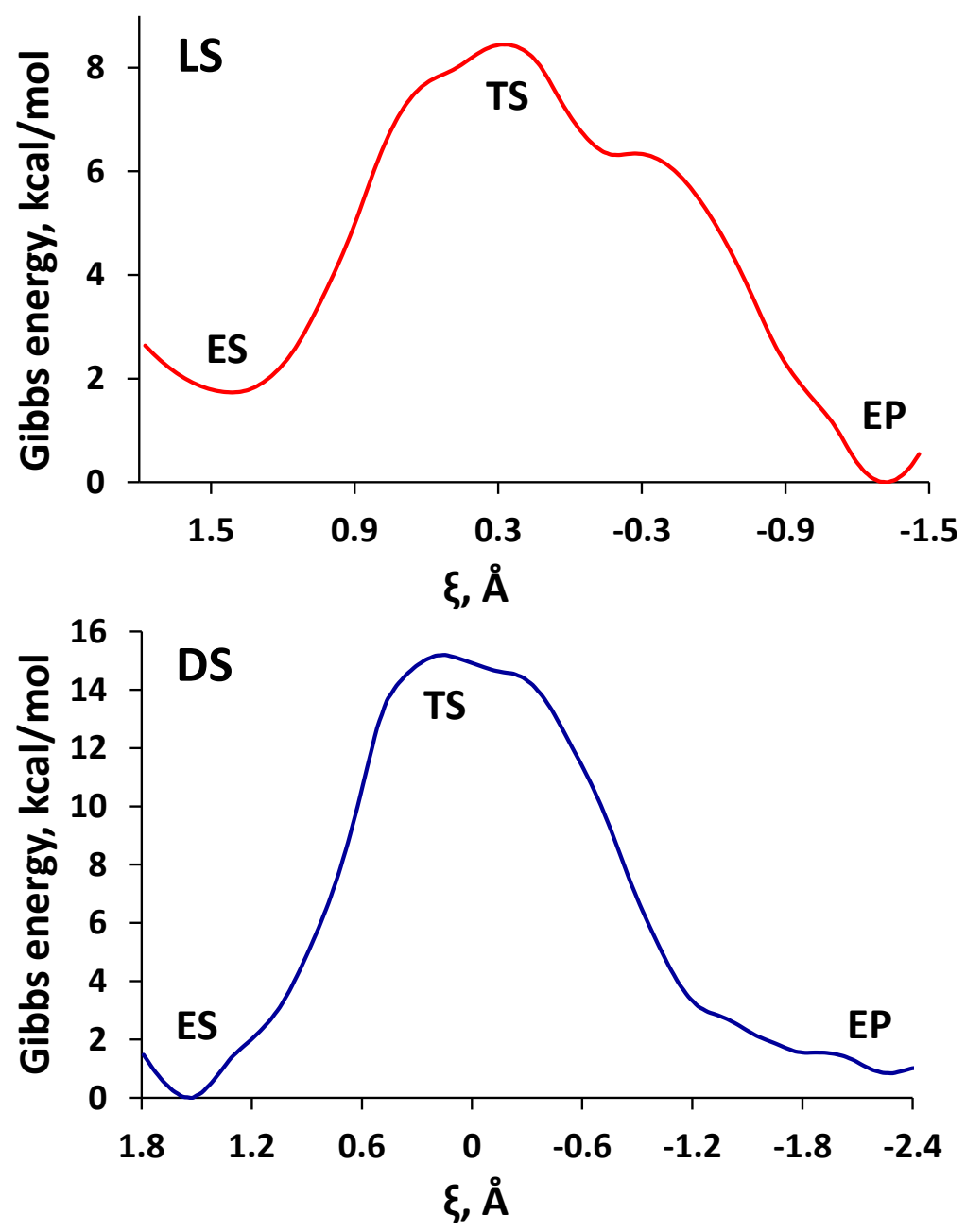

Figure 8. Gibbs energy profiles of the ATP $\rightarrow$ cAMP reaction computed in the QM(DFT $(\omega B$ B $97 X-$ D3/6-31G**))/MM(CHARMM) MD simulations. The upper panel refers to the light state, the bottom panel - to the dark state.

Figure 9 illustrates the mechanism of the ATP $\rightarrow$ cAMP reaction in bPAC revealed in the present simulations. It shares common features with that in the mammalian adenylyl cyclase, ${ }^{32}$ including proton transfer from the ribose $\mathrm{O}^{3} \mathrm{H}^{3}$ group to the nearby aspartic acid via a shuttling water molecule concerted with $\mathrm{P}^{\mathrm{A}}-\mathrm{O}^{3 \mathrm{~A}}$ bond cleavage and $\mathrm{O}^{3^{\prime}}-\mathrm{P}^{\mathrm{A}}$ bond formation. 


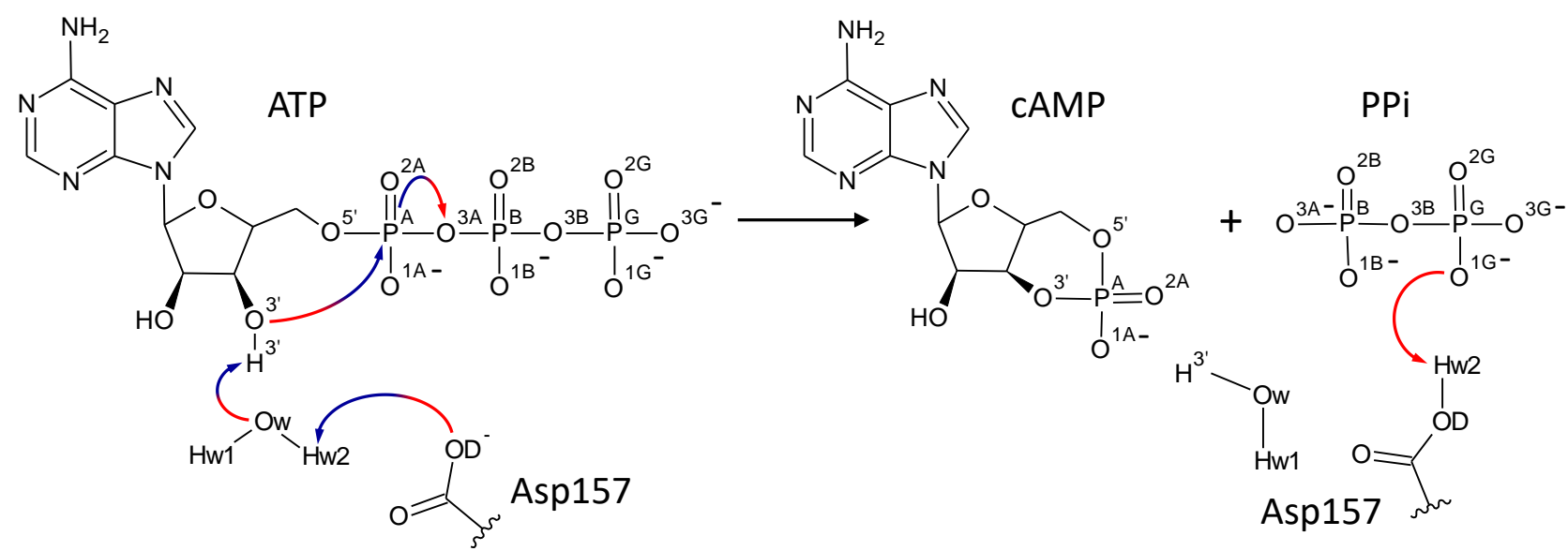

Figure 9. Reaction mechanism of the ATP hydrolysis in the active site of catalytic domain of bPAC. The blue-red arrows depict the processes occurring in both DS and LS. The red arrow in the right part corresponds to the process occurring only in the LS.

We emphasize an important role of switching between conformations with different positions of Arg278 in the dark and light states of the system. In LS, Arg278 forms hydrogen bond with $\mathrm{O}^{1 \mathrm{~A}}$ atom in the reactants and forms additional hydrogen bond with the $\mathrm{O}^{3 \mathrm{~A}}$ atom in the area of TS (transition state) that stabilizes the latter. In DS, Arg278 interacts with the pyrophosphate fragment obtained from the $\beta$ - and $\gamma$-phosphates of the initial ATP molecule. The conformation of Arg278 in the light state also contributes to a lower energy of EP relative to ES by about $2 \mathrm{kcal} / \mathrm{mol}$. The reaction pathway in the dark state does not show EP stabilization relative to ES.

One more role of the Arg278 conformations is in protonation of the pyrophosphate moiety after formation of EP, which should facilitate release of PPi from the active site. In LS, pyrophosphate $\left(\mathrm{P}_{2} \mathrm{O}_{7}{ }^{4-}\right)$ is stabilized by a hydrogen bond with the positively charged Arg284 and by three coordination bonds with the magnesium cations. This configuration facilitates proton transfer from Asp157 to the oxygen atom of the $\gamma$-phosphate group. In DS, the pyrophosphate is stabilized by two additional hydrogen bonds with Arg278 and simultaneous proton transfer does not occur. We attempted to compute the Gibbs energy profile of the proton transfer in the DS; however, we failed to localize a minimum energy point with the proton forming a covalent bond with the pyrophosphate.

It should be noted that here we analyze only the intrinsic chemical reaction ATP $\rightarrow$ cAMP + PPi that proceeds between the ES and EP points inside the protein cavity. The measured steady state reaction rate constant in $\mathrm{bPAC}^{15}$ refer to the complete set of processes including substrate deposition and product release besides the chemical step. Therefore, we can only speculate on the agreement between the experimental and theoretical results. However, what we definitely observe in molecular 
simulations is a considerable lowering of the chemical reaction energy barrier and formation of PPi in the protonation state facilitating its release from the active site.

The significance of conformational changes in the side chain of the arginine residue in the enzyme active site in modulating the rate of the chemical reaction with ATP is not unexpected. The so-called arginine finger is well recognized in reactions of the enzyme-catalyzed phosphorus-oxygen bond cleavage in nucleotide triphosphate (NTP). The catalytically important Arg residue donated from external proteins to the active site can dramatically accelerate chemical reactions. ${ }^{58-64}$ There are known examples of conformational changes in arginine position due to formation of protein complexes which lead to the manifold increase of the NTP hydrolysis rate constant. ${ }^{65}$ These data support the idea proposed in the present study on the role of conformational changes of the side chain of Arg278 as an issue responsible for the acceleration of ATP to cAMP conversion.

\section{Conclusions}

The results of classical and QM/MM MD simulations of the wild type and mutant photoactivated adenylyl cyclase bPAC allow us to explain the mechanism of acceleration of the ATP to $\mathrm{cAMP}$ conversion in the catalytic AC domain after illumination of the photosensing BLUF domain. Following the dynamical network analysis, we recognize the signal transmission pathways from the chromophore environment to the AC active site. The $1 \mu \mathrm{s} \mathrm{MD} \mathrm{trajectories} \mathrm{reveal} \mathrm{the} \mathrm{dynamical} \mathrm{shift}$ between conformations of the side chain of Arg278 near the phosphate groups of ATP in the active site of AC upon transformation from the dark to the light state of the complex. Moreover, the corresponding distribution in arginine conformations in the bPAC-Y7F is intermediate between the dark and light states of the wild-type complex in agreement with the observed intermediate catalytic activity of the mutated form. We compare the Gibbs energy profiles of the chemical reaction computed at the QM(DFT( $\omega$ B97X-D3/6-31G**))/MM(CHARMM) level with the ab initio type potentials in QM in the DS and LS systems. These profiles show a considerable lowering of the activation energy barrier due to the transformation from the dark to the light state, what is qualitatively consistent with the experimentally detected increase of the reaction rate. The decrease of the reaction barrier in the light-activated system is due to a specific position of $\operatorname{Arg} 278$, which stabilizes pentacoordinated phosphorus of the $\alpha$-phosphate group in the transition state. The disclosed mechanism of the reaction $\mathrm{ATP} \rightarrow \mathrm{cAMP}+\mathrm{PPi}$ in the bPAC active site shares common features with that in the mammalian adenylyl cyclase; it includes proton transfer from the ribose $\mathrm{O}^{3} \mathrm{H}^{3}$ group in ATP to the nearby aspartic acid via a shuttling water molecule concerted with $\mathrm{P}^{\mathrm{A}}-\mathrm{O}^{3 \mathrm{~A}}$ bond cleavage and $\mathrm{O}^{3}-\mathrm{P}^{\mathrm{A}}$ bond formation. 
We demonstrate that mapping the Laplacian of the electron density computed for the enzyme-substrate complexes in the $\mathrm{AC}$ active site provides easily visible images of the proper nucleophile orientation that allow us to distinguish between reactive and nonreactive ES structures. In summary, the results of these simulations for the first time describe a consistent picture of the photoinduced and acceleration of the enzymatic reaction in bPAC.

\section{Acknowledgements}

AVN acknowledges support from the Russian Science Foundation (project \#19-73-20032). We acknowledge the use of supercomputer resources of the Joint Supercomputer Center of the Russian Academy of Sciences and the equipment of the shared research facilities of HPC computing resources at Lomonosov Moscow State University. ${ }^{66}$

\section{References}

(1) Losi, A.; Gardner, K. H.; Möglich, A. Blue-Light Receptors for Optogenetics. Chem. Rev. 2018, 118 (21), 10659-10709. https://doi.org/10.1021/acs.chemrev.8b00163.

(2) Schmermund, L.; Jurkaš, V.; Özgen, F. F.; Barone, G. D.; Büchsenschütz, H. C.; Winkler, C. K.; Schmidt, S.; Kourist, R.; Kroutil, W. Photo-Biocatalysis: Biotransformations in the Presence of Light. ACS Catal. 2019, 9 (5), 4115-4144. https://doi.org/10.1021/acscatal.9b00656.

(3) Steuer Costa, W.; Yu, S.; Liewald, J. F.; Gottschalk, A. Fast CAMP Modulation of Neurotransmission via Neuropeptide Signals and Vesicle Loading. Curr. Biol. 2017, 27 (4), 495-507. https://doi.org/10.1016/j.cub.2016.12.055.

(4) Zhang, F.; Tzanakakis, E. S. Optogenetic Regulation of Insulin Secretion in Pancreatic $\beta$-Cells. Sci. Rep. 2017, 7 (1), 9357. https://doi.org/10.1038/s41598-017-09937-0.

(5) Luyben, T. T.; Rai, J.; Li, H.; Georgiou, J.; Avila, A.; Zhen, M.; Collingridge, G. L.; Tominaga, T.; Okamoto, K. Optogenetic Manipulation of Postsynaptic CAMP Using a Novel Transgenic Mouse Line Enables Synaptic Plasticity and Enhances Depolarization Following Tetanic Stimulation in the Hippocampal Dentate Gyrus. Front. Neural Circuits 2020, 14. https://doi.org/10.3389/fncir.2020.00024.

(6) Blackburn, G. M.; Cherfils, J.; Moss, G. P.; Richards, N. G. J.; Waltho, J. P.; Williams, N. H.; Wittinghofer, A. How to Name Atoms in Phosphates, Polyphosphates, Their Derivatives and Mimics, and Transition State Analogues for Enzyme-Catalysed Phosphoryl Transfer Reactions 
(IUPAC Recommendations 2016). Pure Appl. Chem. 2017, 89 (5), 653-675. https://doi.org/10.1515/pac-2016-0202.

(7) Naim, N.; Reece, J. M.; Zhang, X.; Altschuler, D. L. Dual Activation of Production Through Photostimulation or Chemical Stimulation. In Photoswitching Proteins. Methods in Molecular Biology; Niopek, D., Ed.; Humana: New York, NY, 2020; pp 201-216. https://doi.org/10.1007/978-1-0716-0755-8_14.

(8) Khannpnavar, B.; Mehta, V.; Qi, C.; Korkhov, V. Structure and Function of Adenylyl Cyclases, Key Enzymes in Cellular Signaling. Curr. Opin. Struct. Biol. 2020, 63, 34-41. https://doi.org/10.1016/j.sbi.2020.03.003.

(9) Bassler, J.; Schultz, J. E.; Lupas, A. N. Adenylate Cyclases: Receivers, Transducers, and Generators of Signals. Cell. Signal. 2018, 46, 135-144. https://doi.org/10.1016/j.cellsig.2018.03.002.

(10) Iwata, T.; Nagai, T.; Ito, S.; Osoegawa, S.; Iseki, M.; Watanabe, M.; Unno, M.; Kitagawa, S.; Kandori, H. Hydrogen Bonding Environments in the Photocycle Process around the Flavin Chromophore of the AppA-BLUF Domain. J. Am. Chem. Soc. 2018, 140 (38), 11982-11991. https://doi.org/10.1021/jacs.8b05123.

(11) Iseki, M.; Matsunaga, S.; Murakami, A.; Ohno, K.; Shiga, K.; Yoshida, K.; Sugai, M.; Takahashi, T.; Hori, T.; Watanabe, M. A Blue-Light-Activated Adenylyl Cyclase Mediates Photoavoidance in Euglena Gracilis. Nature 2002, 415 (6875), 1047-1051. https://doi.org/10.1038/4151047a.

(12) Ohki, M.; Sato-Tomita, A.; Matsunaga, S.; Iseki, M.; Tame, J. R. H.; Shibayama, N.; Park, S.Y. Molecular Mechanism of Photoactivation of a Light-Regulated Adenylate Cyclase. Proc. Natl. Acad. Sci. 2017, 114 (32), 8562-8567. https://doi.org/10.1073/pnas.1704391114.

(13) Ohki, M.; Sugiyama, K.; Kawai, F.; Tanaka, H.; Nihei, Y.; Unzai, S.; Takebe, M.; Matsunaga, S.; Adachi, S.; Shibayama, N.; Zhou, Z.; Koyama, R.; Ikegaya, Y.; Takahashi, T.; Tame, J. R. H.; Iseki, M.; Park, S.-Y. Structural Insight into Photoactivation of an Adenylate Cyclase from a Photosynthetic Cyanobacterium. Proc. Natl. Acad. Sci. 2016, 113 (24), 6659-6664. https://doi.org/10.1073/pnas.1517520113.

(14) Ryu, M.-H.; Moskvin, O. V.; Siltberg-Liberles, J.; Gomelsky, M. Natural and Engineered Photoactivated Nucleotidyl Cyclases for Optogenetic Applications. J. Biol. Chem. 2010, 285 (53), 41501-41508. https://doi.org/10.1074/jbc.M110.177600.

(15) Lindner, R.; Hartmann, E.; Tarnawski, M.; Winkler, A.; Frey, D.; Reinstein, J.; Meinhart, A.; 
Schlichting, I. Photoactivation Mechanism of a Bacterial Light-Regulated Adenylyl Cyclase. J. Mol. Biol. 2017, 429 (9), 1336-1351. https://doi.org/10.1016/j.jmb.2017.03.020.

(16) Stierl, M.; Stumpf, P.; Udwari, D.; Gueta, R.; Hagedorn, R.; Losi, A.; Gärtner, W.; Petereit, L.; Efetova, M.; Schwarzel, M.; Oertner, T. G.; Nagel, G.; Hegemann, P. Light Modulation of Cellular CAMP by a Small Bacterial Photoactivated Adenylyl Cyclase, BPAC, of the Soil Bacterium Beggiatoa. J. Biol. Chem. 2011, 286 (2), 1181-1188. https://doi.org/10.1074/jbc.M110.185496.

(17) Penzkofer, A.; Stierl, M.; Hegemann, P.; Kateriya, S. Photo-Dynamics of the BLUF Domain Containing Soluble Adenylate Cyclase (NPAC) from the Amoeboflagellate Naegleria Gruberi NEG-M Strain. Chem. Phys. 2011, $387 \quad$ (1-3), 25-38. https://doi.org/10.1016/j.chemphys.2011.05.028.

(18) Blain-Hartung, M.; Rockwell, N. C.; Moreno, M. V.; Martin, S. S.; Gan, F.; Bryant, D. A.; Lagarias, J. C. Cyanobacteriochrome-Based Photoswitchable Adenylyl Cyclases (CPACs) for Broad Spectrum Light Regulation of CAMP Levels in Cells. J. Biol. Chem. 2018, 293 (22), 8473-8483. https://doi.org/10.1074/jbc.RA118.002258.

(19) Scheib, U.; Broser, M.; Constantin, O. M.; Yang, S.; Gao, S.; Mukherjee, S.; Stehfest, K.; Nagel, G.; Gee, C. E.; Hegemann, P. Rhodopsin-Cyclases for Photocontrol of CGMP/CAMP and $2.3 \AA$ Structure of the Adenylyl Cyclase Domain. Nat. Commun. 2018, 9 (1), 2046. https://doi.org/10.1038/s41467-018-04428-w.

(20) Fushimi, K.; Enomoto, G.; Ikeuchi, M.; Narikawa, R. Distinctive Properties of Dark Reversion Kinetics between Two Red/Green-Type Cyanobacteriochromes and Their Application in the Photoregulation of CAMP Synthesis. Photochem. Photobiol. 2017, 93 (3), 681-691. https://doi.org/10.1111/php.12732.

(21) Hirano, M.; Takebe, M.; Ishido, T.; Ide, T.; Matsunaga, S. The C-Terminal Region Affects the Activity of Photoactivated Adenylyl Cyclase from Oscillatoria Acuminata. Sci. Rep. 2019, 9 (1), 20262. https://doi.org/10.1038/s41598-019-56721-3.

(22) Stierl, M.; Penzkofer, A.; Kennis, J. T. M.; Hegemann, P.; Mathes, T. Key Residues for the Light Regulation of the Blue Light-Activated Adenylyl Cyclase from Beggiatoa Sp. Biochemistry 2014, 53 (31), 5121-5130. https://doi.org/10.1021/bi500479v.

(23) Yuan, H.; Dragnea, V.; Wu, Q.; Gardner, K. H.; Bauer, C. E. Mutational and Structural Studies of the PixD BLUF Output Signal That Affects Light-Regulated Interactions with PixE. Biochemistry 2011, 50 (29), 6365-6375. https://doi.org/10.1021/bi200701d. 
(24) Dessauer, C. W.; Gilman, A. G. The Catalytic Mechanism of Mammalian Adenylyl Cyclase. J. Biol. Chem. 1997, 272 (44), 27787-27795. https://doi.org/10.1074/jbc.272.44.27787.

(25) Tesmer, J. J.; Sunahara, R. K.; Gilman, A. G.; Sprang, S. R. Crystal Structure of the Catalytic Domains of Adenylyl Cyclase in a Complex with Gs·GTPS. Science 1997, 278 (5345), 19071916. https://doi.org/10.1126/science.278.5345.1907.

(26) Domratcheva, T.; Grigorenko, B. L.; Schlichting, I.; Nemukhin, A. V. Molecular Models Predict Light-Induced Glutamine Tautomerization in BLUF Photoreceptors. Biophys. J. 2008, 94 (10), 3872-3879. https://doi.org/10.1529/biophysj.107.124172.

(27) Domratcheva, T.; Hartmann, E.; Schlichting, I.; Kottke, T. Evidence for Tautomerisation of Glutamine in BLUF Blue Light Receptors by Vibrational Spectroscopy and Computational Chemistry. Sci. Rep. 2016, 6, 22669. https://doi.org/10.1038/srep22669.

(28) Grigorenko, B. L.; Khrenova, M. G.; Nemukhin, A. V. Amide-Imide Tautomerization in the Glutamine Side Chain in Enzymatic and Photochemical Reactions in Proteins. Phys. Chem. Chem. Phys. 2018, 20 (37), 23827-23836. https://doi.org/10.1039/C8CP04817G.

(29) Khrenova, M. G. G.; Nemukhin, A. V. V.; Domratcheva, T. Photoinduced Electron Transfer Facilitates Tautomerization of the Conserved Signaling Glutamine Side Chain in BLUF Protein Light Sensors. J. Phys. Chem. B 2013, 117 (8), 2369-2377. https://doi.org/10.1021/jp312775x.

(30) Goings, J. J.; Hammes-Schiffer, S. Early Photocycle of Slr1694 Blue-Light Using Flavin Photoreceptor Unraveled through Adiabatic Excited-State Quantum Mechanical/Molecular Mechanical Dynamics. J. Am. Chem. Soc. 2019, 141 (51), 20470-20479. https://doi.org/10.1021/jacs.9b11196.

(31) Goings, J. J.; Li, P.; Zhu, Q.; Hammes-Schiffer, S. Formation of an Unusual Glutamine Tautomer in a Blue Light Using Flavin Photocycle Characterizes the Light-Adapted State. Proc. Natl. Acad. Sci. 2020, 202016719. https://doi.org/10.1073/pnas.2016719117.

(32) Grigorenko, B.; Polyakov, I.; Nemukhin, A. Mechanisms of ATP to CAMP Conversion Catalyzed by the Mammalian Adenylyl Cyclase: A Role of Magnesium Coordination Shells and Proton Wires. J. Phys. Chem. B 2020, 124 (3), 451-460. https://doi.org/10.1021/acs.jpcb.9b07349.

(33) Mones, L.; Tang, W.-J.; Florián, J. Empirical Valence Bond Simulations of the Chemical Mechanism of ATP to CAMP Conversion by Anthrax Edema Factor. Biochemistry 2013, 52 (15), 2672-2682. https://doi.org/10.1021/bi400088y.

(34) Hahn, D. K.; Tusell, J. R.; Sprang, S. R.; Chu, X. Catalytic Mechanism of Mammalian Adenylyl 
Cyclase: A Computational Investigation. Biochemistry 2015, 54 (40), 6252-6262. https://doi.org/10.1021/acs.biochem.5b00655.

(35) Jara, G. E.; Martínez, L. Anthrax Edema Factor: An Ion-Adaptive Mechanism of Catalysis with Increased Transition-State Conformational Flexibility. J. Phys. Chem. B 2016, 120 (27), 65046514. https://doi.org/10.1021/acs.jpcb.6b02527.

(36) Carroll, M. T.; Cheeseman, J. R.; Osman, R.; Weinstein, H. Nucleophilic Addition to Activated Double Bonds: Predictions of Reactivity from the Laplacian of the Charge Density. J. Phys. Chem. 1989, 93 (13), 5120-5123. https://doi.org/10.1021/j100350a019.

(37) Khrenova, M. G.; Tsirelson, V. G.; Nemukhin, A. V. Dynamical Properties of EnzymeSubstrate Complexes Disclose Substrate Specificity of the SARS-CoV-2 Main Protease as Characterized by the Electron Density Descriptors. Phys. Chem. Chem. Phys. 2020, 22 (34), 19069-19079. https://doi.org/10.1039/D0CP03560B.

(38) Khrenova, M. G.; Nemukhin, A. V.; Tsirelson, V. G. Discrimination of Enzyme-Substrate Complexes by Reactivity Using the Electron Density Analysis: Peptide Bond Hydrolysis by the Matrix Metalloproteinase-2. Mendeleev Commun. 2020, 30 (5), 583-585. https://doi.org/10.1016/j.mencom.2020.09.010.

(39) Word, J. M.; Lovell, S. C.; Richardson, J. S.; Richardson, D. C. Asparagine and Glutamine: Using Hydrogen Atom Contacts in the Choice of Side-Chain Amide Orientation. J. Mol. Biol. 1999, 285 (4), 1735-1747. https://doi.org/10.1006/jmbi.1998.2401.

(40) Kleinboelting, S.; van den Heuvel, J.; Steegborn, C. Structural Analysis of Human Soluble Adenylyl Cyclase and Crystal Structures of Its Nucleotide Complexes - Implications for Cyclase Catalysis and Evolution. FEBS J. 2014, 281 (18), 4151-4164. https://doi.org/10.1111/febs.12913.

(41) Best, R. B.; Zhu, X.; Shim, J.; Lopes, P. E. M.; Mittal, J.; Feig, M.; MacKerell, A. D. Optimization of the Additive CHARMM All-Atom Protein Force Field Targeting Improved Sampling of the Backbone $\phi, \psi$ and Side-Chain $\chi_{1}$ and $\chi_{2}$ Dihedral Angles. J. Chem. Theory Comput. 2012, 8 (9), 3257-3273. https://doi.org/10.1021/ct300400x.

(42) Denning, E. J.; Priyakumar, U. D.; Nilsson, L.; Mackerell, A. D. Impact of 2'-Hydroxyl Sampling on the Conformational Properties of RNA: Update of the CHARMM All-Atom Additive Force Field for RNA. J. Comput. Chem. 2011, 32 (9), 1929-1943. https://doi.org/10.1002/jcc.21777.

(43) Jorgensen, W. L.; Chandrasekhar, J.; Madura, J. D.; Impey, R. W.; Klein, M. L. Comparison 
of Simple Potential Functions for Simulating Liquid Water. J. Chem. Phys. 1983, 79 (2), 926935. https://doi.org/10.1063/1.445869.

(44) Vanommeslaeghe, K.; Hatcher, E.; Acharya, C.; Kundu, S.; Zhong, S.; Shim, J.; Darian, E.; Guvench, O.; Lopes, P.; Vorobyov, I.; Mackerell, A. D. CHARMM General Force Field (CGenFF): A Force Field for Drug-like Molecules Compatible with the CHARMM All-Atom Additive Biological Force Fields. J. Comput. Chem. 2010, 31 (4), 671-690. https://doi.org/10.1002/jcc.21367.

(45) Khrenova, M. G.; Meteleshko, Y. I.; Nemukhin, A. V. Mutants of the Flavoprotein ILOV as Prospective Red-Shifted Fluorescent Markers. J. Phys. Chem. B 2017, 121 (43), 10018-10025. https://doi.org/10.1021/acs.jpcb.7b07533.

(46) Khrenova, M. G. M. G.; Domratcheva, T.; Schlichting, I.; Grigorenko, B. L.; Nemukhin, A. V. A. V. Computational Characterization of Reaction Intermediates in the Photocycle of the Sensory Domain of the AppA Blue Light Photoreceptor. Photochem. Photobiol. 2011, 87 (3), 564-573. https://doi.org/10.1111/j.1751-1097.2010.00861.x.

(47) Humphrey, W.; Dalke, A.; Schulten, K. VMD: Visual Molecular Dynamics. J. Mol. Graph. 1996, 14 (1), 33-38. https://doi.org/10.1016/0263-7855(96)00018-5.

(48) Phillips, J. C.; Braun, R.; Wang, W.; Gumbart, J.; Tajkhorshid, E.; Villa, E.; Chipot, C.; Skeel, R. D.; Kalé, L.; Schulten, K. Scalable Molecular Dynamics with NAMD. J. Comput. Chem. 2005, 26 (16), 1781-1802. https://doi.org/10.1002/jcc.20289.

(49) Kots, E. D.; Khrenova, M. G.; Nemukhin, A. V. Allosteric Control of N-Acetyl-Aspartate Hydrolysis by the Y231C and F295S Mutants of Human Aspartoacylase. J. Chem. Inf. Model. 2019, 59 (5), 2299-2308. https://doi.org/10.1021/acs.jcim.8b00666.

(50) Sethi, A.; Eargle, J.; Black, A. A.; Luthey-Schulten, Z. Dynamical Networks in TRNA:Protein Complexes. Proc. Natl. Acad. Sci. 2009, $106 \quad$ (16), 6620-6625. https://doi.org/10.1073/pnas.0810961106.

(51) Glykos, N. M. Software News and Updates Carma: A Molecular Dynamics Analysis Program. J. Comput. Chem. 2006, 27 (14), 1765-1768. https://doi.org/10.1002/jcc.20482.

(52) Melo, M. C. R.; Bernardi, R. C.; Rudack, T.; Scheurer, M.; Riplinger, C.; Phillips, J. C.; Maia, J. D. C.; Rocha, G. B.; Ribeiro, J. V; Stone, J. E.; Neese, F.; Schulten, K.; Luthey-Schulten, Z. NAMD Goes Quantum: An Integrative Suite for Hybrid Simulations. Nat. Methods 2018, 15 (5), 351-354. https://doi.org/10.1038/nmeth.4638.

(53) Seritan, S.; Bannwarth, C.; Fales, B. S.; Hohenstein, E. G.; Isborn, C. M.; Kokkila-Schumacher, 
S. I. L.; Li, X.; Liu, F.; Luehr, N.; Snyder, J. W.; Song, C.; Titov, A. V.; Ufimtsev, I. S.; Wang, L.; Martínez, T. J. TeraChem : A Graphical Processing Unit-accelerated Electronic Structure Package for Large-scale Ab Initio Molecular Dynamics. WIREs Comput. Mol. Sci. 2020. https://doi.org/10.1002/wcms.1494.

(54) Matta, C. F.; Bader, R. F. W. Atoms-in-Molecules Study of the Genetically Encoded Amino Acids. III. Bond and Atomic Properties and Their Correlations with Experiment Including Mutation-Induced Changes in Protein Stability and Genetic Coding. Proteins Struct. Funct. Bioinforma. 2003, 52 (3), 360-399. https://doi.org/10.1002/prot.10414.

(55) Khrenova, M. G.; Kulakova, A. M.; Nemukhin, A. V. Proof of Concept for Poor Inhibitor Binding and Efficient Formation of Covalent Adducts of KRAS G12C and ARS Compounds. Org. Biomol. Chem. 2020, 18 (16), 3069-3081. https://doi.org/10.1039/D0OB00071J.

(56) Khrenova, M. G.; Krivitskaya, A. V.; Tsirelson, V. G. The QM/MM-QTAIM Approach Reveals the Nature of the Different Reactivity of Cephalosporins in the Active Site of L1 Metallo- $\beta$-Lactamase. New J. Chem. 2019, $43 \quad$ (19), 7329-7338. https://doi.org/10.1039/C9NJ00254E.

(57) Lu, T.; Chen, F. Multiwfn: A Multifunctional Wavefunction Analyzer. J. Comput. Chem. 2012, 33 (5), 580-592. https://doi.org/10.1002/jcc.22885.

(58) Nagy, G. N.; Suardíaz, R.; Lopata, A.; Ozohanics, O.; Vékey, K.; Brooks, B. R.; Leveles, I.; Tóth, J.; Vértessy, B. G.; Rosta, E. Structural Characterization of Arginine Fingers: Identification of an Arginine Finger for the Pyrophosphatase DUTPases. J. Am. Chem. Soc. 2016, 138 (45), 15035-15045. https://doi.org/10.1021/jacs.6b09012.

(59) Hayashi, S.; Ueno, H.; Shaikh, A. R.; Umemura, M.; Kamiya, M.; Ito, Y.; Ikeguchi, M.; Komoriya, Y.; Iino, R.; Noji, H. Molecular Mechanism of ATP Hydrolysis in F 1 -ATPase Revealed by Molecular Simulations and Single-Molecule Observations. J. Am. Chem. Soc. 2012, 134 (20), 8447-8454. https://doi.org/10.1021/ja211027m.

(60) Rydzek, S.; Shein, M.; Bielytskyi, P.; Schütz, A. K. Observation of a Transient Reaction Intermediate Illuminates the Mechanochemical Cycle of the AAA-ATPase P97. J. Am. Chem. Soc. 2020, 142 (34), 14472-14480. https://doi.org/10.1021/jacs.0c03180.

(61) Hanson, P. I.; Whiteheart, S. W. AAA+ Proteins: Have Engine, Will Work. Nat. Rev. Mol. Cell Biol. 2005, 6 (7), 519-529. https://doi.org/10.1038/nrm1684.

(62) Gasper, R.; Meyer, S.; Gotthardt, K.; Sirajuddin, M.; Wittinghofer, A. It Takes Two to Tango: Regulation of G Proteins by Dimerization. Nat. Rev. Mol. Cell Biol. 2009, 10 (6), 423-429. 
https://doi.org/10.1038/nrm2689.

(63) Calixto, A. R.; Moreira, C.; Pabis, A.; Kötting, C.; Gerwert, K.; Rudack, T.; Kamerlin, S. C. L. GTP Hydrolysis Without an Active Site Base: A Unifying Mechanism for Ras and Related GTPases. J. Am. Chem. Soc. 2019, $141 \quad$ (27), 10684-10701. https://doi.org/10.1021/jacs.9b03193.

(64) Ahmadian, M. R.; Stege, P.; Scheffzek, K.; Wittinghofer, A. Confirmation of the ArginineFinger Hypothesis for the GAP-Stimulated GTP-Hydrolysis Reaction of Ras. Nat. Struct. Biol. 1997, 4 (9), 686-689. https://doi.org/10.1038/nsb0997-686.

(65) Mann, D.; Teuber, C.; Tennigkeit, S. A.; Schröter, G.; Gerwert, K.; Kötting, C. Mechanism of the Intrinsic Arginine Finger in Heterotrimeric G Proteins. Proc. Natl. Acad. Sci. 2016, 113 (50), E8041-E8050. https://doi.org/10.1073/pnas.1612394113.

(66) Voevodin, V. V.; Antonov, A. S.; Nikitenko, D. A.; Shvets, P. A.; Sobolev, S. I.; Sidorov, I. Y.; Stefanov, K. S.; Voevodin, V. V.; Zhumatiy, S. A. Supercomputer Lomonosov-2: Large Scale, Deep Monitoring and Fine Analytics for the User Community. Supercomput. Front. Innov. 2019, 6 (2), 4-11. https://doi.org/10.14529/jsfi190201. 